\title{
Research on the Interactive Problems and Countermeasures of Food Intelligent Packaging in the Post Epidemic Era
}

\author{
Juzheng Zhang ${ }^{1, \dagger}$, Qiutong $\mathrm{Wu}^{2, \dagger}$, Ruikun Chen ${ }^{3, \dagger}$ \\ ${ }^{1}$ Juzheng Zhang: School of Management, Henan University of Science and Technology, Email:2489684930@qq.com \\ ${ }^{2}$ Qiutong Wu: Leeds University Business School, University of Leeds, Email:Akillen920@ gmail.com \\ ${ }^{3}$ Ruikun Chen:Rachel Carson college, University of California, Santa Cruz, Email:rchen91@ucsc.edu \\ *Corresponding author. Email: rchen91@ucsc.edu \\ tThese authors contributed equally.
}

\begin{abstract}
Intelligent packaging is one of the main technologies that achieve to make our lives more convenient in recent decades. People are paying attention to the safeness that packaging can offer, especially during the pandemic. This paper collected and analyzed the research results of intelligent packaging in the field of food in recent years through journals, network reports and other channels before the research. We found several ways of interacting, for such as QR codes, RFID tags and smartphones. Regardless of the progress in the area of food intelligent packaging, some problems appeared, such as the high costs and simplification of the methods. The final discussion states that the interaction way could be more diverse, or more technologies could be used to improve packaging' s efficiency. Further researches and experiments may need in order to fulfill the application of future technologies and progress.
\end{abstract}

Keywords: Food intelligent packaging, Post Epidemic Era, interactive

\section{INTRODUCTION}

The outbreak of novel coronavirus pneumonia has brought significant challenges to the world's economy, culture and life. With the normalization of the epidemic situation, people's attention to safety and health has reached an unprecedented peak in modern times. At present, although novel coronavirus pneumonia has been initially controlled, the spread of the virus is still very alarming to people all over the world. New coronavirus can be attached to the object's surface, through contact, in the way of "thing to person" to cause new transmission. As an essential part of people's lives and an essential component of the commodity economy, food packaging has attracted people's attention. People hope that food packaging can meet the people's current needs for safety, health, sterilization, prevention and control of the epidemic situation. As the latest packaging development form in the market, the intelligent packaging advantage fully meet the people's needs in the above aspects.

As a new packaging form, intelligent packaging is the product of the combination of people's needs and technology in the new era. It meets the needs of modern people for packaging and gives new added value to commodities. This added value is often reflected by the interactive elements in the intelligent packaging. The commodity shows the value of "own" to the customers through the intelligent packaging outside it. The interactive intelligent packaging not only breaks the "communication barrier" between the goods and customers in the traditional sense and satisfies the customer's satisfaction of "emotional needs" under the premise of meeting the customer needs. When applied in the food field, the intelligent packaging can display the origin, production composition, logistics situation of food by technical means, and even keep the freshness and simulated smell.

In the post epidemic era, the application of food intelligent packaging technology can fully meet people's food safety and health needs. Intelligent food packaging can ensure food safety inside the packaging through the application of antibacterial technology and sterilization technology. People can search for any information they want about products on their smart phones through QR codes, electronic tags and so on. VR technology and 
virtual technology can make customers "touch" the food and observe the food's actual situations.

In this paper, we focus on interactive intelligent packaging in the field of food. One of the most important characteristics of intelligent packaging is its interactive performance as new technology industry. This kind of interaction can establish a kind of contact between customers and goods that can meet customers' emotional needs, and enhance customers' purchase intention through the interaction between people and goods. People can scan the code and other forms, from simply accepting the text information on the food packaging in the past, to now judging the actual value of the commodity through graphics, evaluation of the buyer and other information. This kind of interactive embodiment also helps enterprises to improve sales volume and establish loyal customer groups, which has significant economic value. Commonly, customers can get the commodity information and instructions by scanning the code and other means. Enterprises can reduce customers' awareness of preparedness" , increase economic benefits and improve corporate image through this unique interaction of intelligent packaging. For example, Treasury Wine Estates, Australia's largest wine group is also the first wine company to introduce AR technology into wine labels. It once launched a wine called "19 crimes wine". When customers scan the labels, the criminals on the labels will "resurrect" and tell you their stories. According to the company, this kind of red wine's sales results increased the company's sales volume by $60 \%$ and sales by $70 \%$.

\section{LITERATURE REVIEW}

With the development of the times, the concept of intelligence has been widely used in people's daily life. Compared with the traditional food packaging design, the application and development of new intelligent packaging technology have tended to a new height. As the basis of intelligent packaging, the development of packaging technology determines the development of intelligent packaging to a large extent. Li described the current situation of food packaging in China, analyzed the connotation and control technology of food intelligent packaging in detail, and provided corresponding suggestions for applying these technologies in the 5G era [1]. Zhu Y et al. analyzed the material, structure, technology and other characteristics of intelligent food packaging, described the application and specific principle of intelligent packaging in the field of food preservation, and described its development potential [2]. Chen $\mathrm{K}$ and $\mathrm{Chen} \mathrm{G}$ divided the key technologies of intelligent packaging into intelligent packaging architecture, digital packaging technology, packaging visualization technology and intelligent packaging cloud platform (big data platform) from building the overall technical scheme of intelligent packaging. They introduced the representative technologies and corresponding functions [3]. According to the classification of traditional intelligent packaging, Wang $\mathrm{Y}$ et al. introduced the main application technologies of China's food intelligent packaging market from three aspects of material type, structural type, and information type, and described in detail the development of food intelligent packaging at home and abroad [4]. Since entering the new century, intelligent packaging represented by high-tech technology has developed rapidly. One of the main reasons is the breakthrough of key technology. However, we also need to note that some intelligent packaging technologies restrict the development, such as chips and materials. The content shown in Figure 1 is a kind of food intelligent packaging applied to color-changing materials.

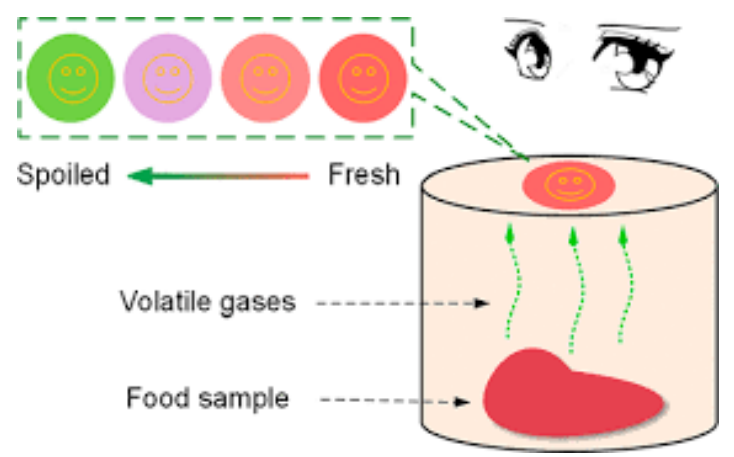

Fig.1 Intelligent color changing food packaging

The main reason why intelligent packaging technology is so popular in the market is that it can improve relationships with customers, thereby indirectly increasing the sales volume of goods and increasing profits. The communication channel is mainly through "interactivity". Zhou R described the inevitable trend of the blend of interactivity and intelligence in the field of food intelligent packaging in detail and introduced a design method and principle of interactive intelligent packaging in food [5]. Shen L et al. described the role of food intelligent packaging label in food supply chain based on the summary of food intelligent packaging label and considered that it could further strengthen its unique data interaction through visualization means, and give full play to its advantages such as quality control, safety protection and cost reduction [6]. Hu Zhicai and Ke Shenghai summarized and classified the color-changing materials in intelligent packaging and believed that the application of color-changing materials in the field of food packaging could better help customers judge the freshness of food and avoid waste [7].

To sum up, we can see that most of the relevant scholars in China focus on technology and application and think that intelligent packaging has very important application significance in the field of food. Simultaneously, the vast majority of research results also reflect the leadership role of interaction in intelligent packaging and put forward constructive suggestions. 
However, we need to note that in the era of normalization of the epidemic situation, people's attention to food safety has reached an unprecedented level, which brings new requirements and challenges to food intelligent packaging. How to make the intelligent packaging meet the requirements of food safety in the new era is the subject of this paper.

\section{RESULT}

\subsection{Excessive costs}

As a newly sprouted thing, intelligent packaging has two sides. In recent years, thanks to a number of breakthroughs in science and technology, smart packaging has also become a "shining star" in the food industry. The new packaging has both positive and negative aspects. Advantages do exist, and however negative effects occurred as we analyzed. A number of costs was added as a new functionality was created. Food producers or packaging manufacturers will need to replace the existing production equipment, train new staff and purchase new production materials. Based on research in the Chinese market, we have found that the temperature indicator currently available for food packaging is around $1 \mathrm{CNY}$ per unit. The RFID tag is around $2 \mathrm{CNY}$ per unit, which is the market price of most small retail goods. Such high packaging costs can lead to an over-concentration of smart food packaging in mid- to high-end products, such as wine, high-end meat, seasonal fruit. Due to the high cost of smart food packaging, it puts huge economic pressure on food manufacturers and retailers who use smart packaging, and they are unsure whether the increased profit from using smart packaging can outweigh or offset this increased cost, which also seriously inhibits the application and development of smart packaging in low-priced food products [8].

What's more, the vast majority of intelligent packaging requires big data platforms or networking agencies need to collect, analyze, process, and filter data and other operations, the establishment and maintenance of online platforms, the hiring of professional staff, which creates additional costs for companies.

\subsection{Monotonous form of interaction}

When mentioned the Intelligent food packaging, "Code scanning" became the most people's first impression. By scanning the OR code on food packaging, users can know the source of food, ingredients, suitability, and other information, which is currently one of the most important forms of intelligent packaging. However, this phenomenon also reflects one of the main drawbacks of intelligent food packaging: the single form of expression. Although various forms of expression for food smart packaging, such as built-in chips, electronic tags, sensors, optical signals, the interaction between the customer and the product are not always the same. However, the form of interaction between the customer and the product has not been developed significantly.Through the act of "scanning", the customer often unilateral access to information and did not form a two-way interaction between the two. With the further development of the commodity economy, the customer's demand for food has changed fundamentally. The customers are paying more attention to the additional value that food brings to them. Therefore, a single, repetitive "communication" way may cause a communication channel that might be too simple, or even form an obstruction [9].

Intelligent packaging at present is overly dependent on phones. Without smartphones or corresponding software, customers will be able to judge whether the product meets their needs through the food's outer packaging, which is not interactive and does not reflect the intelligence of smart packaging. Also, due to the rapid development of society, there will inevitably be "network refugees" (i.e., people who do not know to operate the corresponding smart devices, mainly the elderly group), how to make these people enjoy the convenience and intelligence of smart packaging is becoming one of the main obstacles to the development of intelligent food packaging.

Besides, despite the breakthroughs in new ICT and VR technologies in recent years, there are still not too many unified standards and completed regulations regarding the combination of new technologies and intelligent food packaging since those technologies are still new.

\subsection{Relatively homogeneous in functionality}

As an upgraded system to traditional packaging, the purpose of intelligent packaging services is to improve and reflect the original value of the goods and add more additional value, also creating a new function. Smart packaging for food products currently on the market tends to focus more on the embodiment of a single function, which avoids competition from similar products and enables cost reduction and beautification of appearance.

However, with the normalization of the Covid-19 pandemic today, customers are more focusing on the safety of food at the same time other factors about the goods (e.g., logistics, food ingredients, adverse reaction), which requires a food packaging that can fulfil multiple functions at the same time. As a simple example, it is possible to provide customers with short videos of the whole process of manufacturing and transporting goods while ensuring food safety. According to existing research, smart packaging is mainly divided into functional smart packaging (freshness preservation, temperature control, humidity regulation, mildew prevention), structural smart packaging (safety, 
automation), and information smart packaging (anticounterfeiting, information recording, information display). The existing mainstream food smart packaging can often reflect on one or two of these functions. The lack of functionality will inevitably affect to customers' willingness to buy.

\section{DISCUSSION}

\subsection{Lower the costs}

Cost plays a vital role as an important indicator of the economic efficiency of an enterprise or industry. The main reason why the new intelligent packaging is far more expensive than traditional packaging is intelligent packaging requirements in terms of materials, technology, and production processes. Any progress in science and technology is inseparable from the progress of basic materials, the current smart packaging application materials are mostly synthetic materials, due to the complexity of its process, the price is high, so it needs to continue to stabilize or strengthen the research and development of basic materials. "Chip", as a hot keyword in recent years, also plays a very important role in the intelligent packaging of food products. The cuttingedge nature of technologies such as microchips and printing chips also further increases the cost. In addition, as a new industry, due to the imperfection of production supporting technology, it is not possible to maximize production efficiency, and it is difficult to bring down the cost.

To conclude, to reduce the cost of intelligent food packaging. It is necessary to adhere to the basic materials and application technology research and development as a breakthrough point, to tend to improve the market competition mechanism as the driving force, to go to the cost of promoting profits, the formation of a virtuous circle of development, to be able to fundamentally reduce costs and improve market competitiveness.

\subsection{Adoption of interaction concepts}

In the 21 st century, major scientific and technological breakthroughs have been made, and people's lives have begun to transition from "informatization" to "wisdom" and "intelligence". The development of technologies such as 5G, blockchain, VR, artificial intelligence, and the Internet of Things has also promoted intelligent packaging.

This paper argues that the combination of smart technology and smart packaging can maximize the strong interactivity unique to new smart packaging. For instance, by applying the Internet of Things to food safety, customers can get the information they want in the form of a scan code or input, fully trusting in the product's safety due to the unique connectivity and confidentiality of the Internet of Things. Using VR or artificial intelligence to intelligent food packaging allows customers to move away from passive information browsing and interact with each other in the form of 'conversations' or 'exchanges', improving from simple information browsing to emotional interaction [10].

Moutai of China has launched an Internet of Thingsbased sensory package that helps customers authenticate goods through its built-in chip's anti-counterfeit traceability. Customers can also scan the code to connect to the IoT and find information on production, transportation, or storage on any given day. Moreover, the packaging is also VR-enabled, allowing customers to experience a wide range of applet content by simply scanning it with their mobile phone camera.

In addition, the application of multiple new technologies can also enrich the interactive format of the food smart packaging, moving away from the current more simplex form of interaction.

\subsection{Integration of functions}

During coronavirus, there is no doubt about the importance attached to food safety. Hence, the demand for antimicrobial packaging, aseptic packaging, and other similar food smart packaging has reached its peak. However, the mainstream antimicrobial packaging is currently available on the international market is simply the application of antimicrobial materials made into the outer packaging or placed inside the packaging, making it difficult for consumers to visually observe the degree of antimicrobial or safety. Therefore, this paper proposes to combine this old antimicrobial food packaging with new information technology, which retains the advantages of the old packaging and further 'visualizes' the degree of antimicrobial activity by changing the color of the label, the brightness and the color. The packaging can also be further applied to exported food, medical appliances and pharmaceuticals, having promising market development prospects.

\section{CONCLUSION}

In this paper, through a systematic review of the relevant literature, combined with the novel coronavirus pneumonia epidemic in recent years, we analyzed and summarized the importance of food safety. We finally get the conclusion that food intelligent packaging has great potential in the future. In addition, through the literature review and market research, this paper analyzes the main reasons for the intelligent packaging market's current development. Through the interactive concept design, the development and application of new information technology, and the development of new interactive methods, this paper puts forward the defects of the current food intelligent packaging and puts forward the corresponding optimization strategies. 
Due to the normalization of the epidemic situation, we cannot normally research relevant enterprises and markets. Therefore, we have found and summarized the problems through literature analysis. In addition, this paper is purely from the perspective of management analysis, does not involve technology research and development, industrial design, medical and health care. However, these factors play an important role in the development of food intelligent packaging. Therefore, we will further explore the influence of these factors on developing food intelligent packaging in the follow-up research process. Through the following figure 2 , we can see a predict with the intelligent packaging market in 2025.

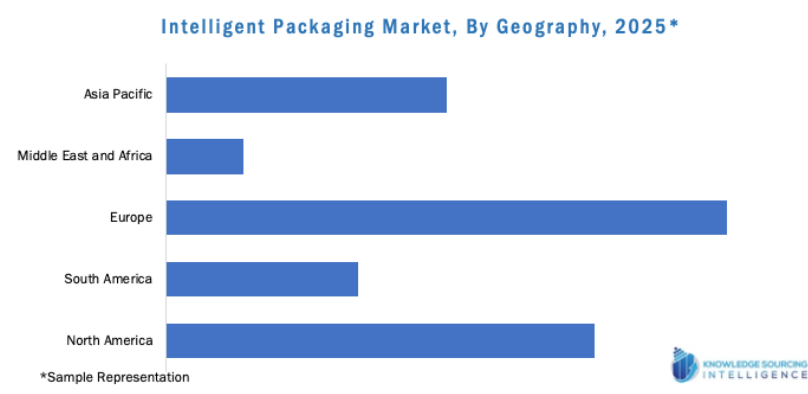

Fig.2 Food intelligent packaging prediction

\section{REFERENCES}

[1] Li Y. Intelligent Control Design of Food Packaging Safety in $5 \mathrm{G}$ Era [J]. Journal of Hunan University of Technology (Social Science Edition), 2020,25(3):815.

[2] Zhu Yong, Hu C, Wang Z. Application of Intelligent Packaging Technology in Food Preservation[J]. Food Science,2007,28(6):356-359.[3]Ｃ. Baier, J-P. Katoen, Principles of Model Checking, MIT Press, 2008.

[3] Chen K, Chen G. Intelligent Packaging:Development Status, Key Technologies and Application Prospects [J]. Packaging Journal, 2019,11(01):1-17,105.

[4] Wang Y, Wang G, Wang Y. Research Progress of Food Intelligent Packaging Technology [J]. Packaging Engineering, 2018,39(11):6-12.

[5] Zhou R. The Interactive Application Of Intelligent Packaging In Food Packaging $[\mathrm{J}]$. Design, 2017(08):128-129.

[6] Shen L, XU Y, Zhan J, Zhu Y, Wang J, Wang H. Application and research on intelligent labels in the food packaging $[\mathrm{J}]$. Science and Technology of Food Industry,2015,36(05):377-383.

[7] $\mathrm{Hu} \mathrm{Z}, \mathrm{Ke} \mathrm{S}$. Application of Intelligent Colorchanging Materials to Packaging and Their Design
Form[J]. Packaging Engineering,2020,41(09):117123.

[8] Zhao D, Zhong C, Zhu L, Xia Z. Development Status, Tendency and Application Prospect of Intelligent Packaging[J].

Packaging engineering,2020,41(13):72-81.

[9] Tang Z. Review and Outlook of Packaging Design Evaluation $[\mathrm{J}]$. Packaging Engineering,2019,40(10):52-58.

[10] Cheng Y. Application Trend of Augmented Reality in Intelligent Packaging[J].Packaging Engineering,2018,39(07):27-30. 